\title{
Analysis of Product Sampling for New Product Diffusion Incorporating Multiple-Unit Ownership
}

\author{
Zhineng Hu, Yurong Pei, and Ruikun Xie \\ Uncertainty Decision-Making Laboratory, Sichuan University, Chengdu 610064, China \\ Correspondence should be addressed to Zhineng Hu; huzn@scu.edu.cn
}

Received 13 January 2014; Accepted 26 March 2014; Published 24 April 2014

Academic Editor: Qun Lin

Copyright ( 2014 Zhineng Hu et al. This is an open access article distributed under the Creative Commons Attribution License, which permits unrestricted use, distribution, and reproduction in any medium, provided the original work is properly cited.

\begin{abstract}
Multiple-unit ownership of nondurable products is an important component of sales in many product categories. Based on the Bass model, this paper develops a new model considering the multiple-unit adoptions as a diffusion process under the influence of product sampling. Though the analysis aims to determine the optimal dynamic sampling effort for a firm and the results demonstrate that experience sampling can accelerate the diffusion process, the best time to send free samples is just before the product being launched. Multiple-unit purchasing behavior can increase sales to make more profit for a firm, and it needs more samples to make the product known much better. The local sensitivity analysis shows that the increase of both external coefficients and internal coefficients has a negative influence on the sampling level, but the internal influence on the subsequent multiple-unit adoptions has little significant influence on the sampling. Using the logistic regression along with linear regression, the global sensitivity analysis gives a whole analysis of the interaction of all factors, which manifests the external influence and multiunit purchase rate are two most important factors to influence the sampling level and net present value of the new product, and presents a two-stage method to determine the sampling level.
\end{abstract}

\section{Introduction}

Since the new product diffusion model by Bass [1], diffusion models have played a major role in marketing science for three decades now (Mahajan et al. [2], Peres et al. [3]). The accepted application of these models for consumer durable products uses the diffusion model to account for the first purchase and the remaining sales by a household (Ratchford et al. [4]). Multiple-unit ownership is common in the purchase process, such as shampoo, washing powder, and toilet paper; however, the accepted approach is incomplete. For these products, a model of multiple-unit ownership is an important component of a manager's toolbox for overall sales trends. More importantly, knowledge of the multiple component trend is valuable for developing product and marketing strategies since user requirements for additional units are often quite different than for the first-purchase units. Bayus et al. [5] proposed one such model of multiple purchases with an age-based formulation similar to replacement models, but their model was developed to forecast the short-term sales of color television which is a durable product. Steffens
[6] pointed out that the sales of multiple-unit adoptions for televisions have been higher than those of both first adopts and replacement purchase since 1977, while for automobiles, they have represented more than $20 \%$ of sales since 1966 in Australia. All of them focus on the durable products, but limited attention is paid on the nondurable products. The development of multiple purchase sales models is in its infancy.

When a new product is launched into market, managers want to know the sales and consumer acceptance rate of the new product in the market (Wang et al. [7]), but consumers may not effectively identify the product's new features; the best way to demonstrate a product's superiority is to get the customer to try it. According to a survey conducted, $75 \%$ of the major corporations in the survey use free samples as a promotional tool for their new products and 52\% use it for their established products. Jain et al. [8] firstly introduced the sampling variable into the Bass model to study the diffusion of innovation, and they pointed that sampling is not only free but has a cost, sampling too little cannot get the expected effect and offering too many free samples is 
a waste of a firm's resources; an appropriate sampling level is very important. Lammers [9] pointed out that sampling is an effective way of promotion and it can enhance the sales volume in a short time, keeps customer loyalty, and encourages consumers to purchase and repeat purchase, and it is highlight in fast moving consumer goods. Hu et al. [10] and $\mathrm{Hu}$ [11] pointed out that free samples can promote the diffusion of new product and also analyzed the influence of individual product diffusion caused by free samples under different price strategies.

In reality, sampling has two effects, according to Heiman et al. [12], the first is the short-term effect, which reflects the change in the probability of a consumer purchasing a product immediately after having being sent free samples, and the second is the long-term effect, which increases the consumers cumulative goodwill formation. In the practical marketing, there are several common cases for product sampling: (1) sampling for experience, for example, a firm usually gives a small bottle/bag of perfume to a customer for experience; (2) sampling for multiple-unit purchase, the firm would also like to give a free one if multiunits were purchased at a time, such as the sale of toothpaste, which may not be considered as free sample in some literature; (3) sampling for bundling sale, the firm would like to sell a bag of toothpaste and toothbrush, whose price is slightly lower than the price of independent sale of toothpaste and toothbrush, but would not like to give a free sample of a bag of toothpaste together with a toothbrush. Sometimes, the firm, however, would like to give a free sample of toothbrush for multiple-unit toothpaste purchase. For these cases, this paper only considers the first case.

Above all, most previous researches are based on the durable product, and product sampling plays an important role in the product diffusion. Comparing to the individual adoption case, this paper establishes an optimization model by considering the multiple-unit ownership as a diffusion process and incorporating the impacts of product sampling and mainly analyzes the product sampling level; specifically, the paper makes a comparison analysis of product diffusion under multiple-unit purchase and individual purchase and then makes a sensitive analysis to find the relationship between the factors and sampling level for giving some significant meanings of marketing management. The rest of the paper is organized as follows. Section 2 states the problem and presents the assumptions and basic model. Section 3 presents the numerical simulation computation for the sampling level and Section 4 gives a sensitive analysis of those factors. Section 5 makes the conclusion and suggestions for further research and the underlying approach.

\section{Model Building}

Combining the individual purchase with the multiple-unit ownership of new product, this section presents a statement about the problem at first and then establishes an optimization diffusion model based on Bass model by considering the impact of sampling.

2.1. Problem Statement. Li and Jin [13] said that the essence of a new products diffusion process is the product information exchanged by which one individual communicates a new product to one or several others. The phenomena of individual adoption of new product and its diffusion through a population have received much attention, but research within the diffusion paradigm that explicitly considers multipleunit ownership of nondurable products is almost nonexistent. For products where multiple-unit ownership is common, especially for the household, the implicit assumption that all purchases are individual adoptions clearly diminishes over time. So the group of adopters can be divided into two kinds: the ones who just purchase one product each time and the others do multiple purchases; the diffusion pattern of individual purchase and multiunit purchase is shown in Figure 1. Thus, in the whole process of diffusion, the consumers divided into two kinds: buyers and unknown consumers. Some unknown consumers become buyers after being impacted by the external influence (e.g., advertisement); other unknown consumers will buy the product due to the internal influence (e.g., oral communication).

Although sampling is an effective way for a firm to increase sales, the use of free samples mainly focuses on the following products. (1) Products, which allow consumers to experience the significant difference, should be easily drawn the distinction by the consumers contacting the products, such as beverages and biscuits. (2) Low-value consumable products, compared to expensive products (such as electrical appliances, whose costs are so high that they are not reasonable as free samples), deserve to be sampled.

Based on the analysis above, some assumptions for the model are presented as follows.

(1) Diffusion process of a new product is independent of other new diffusions.

(2) The geographic boundaries of the social system do not change over the diffusion process.

(3) Nature of a product does not change with the time.

(4) There are no supply restrictions.

(5) The diffusion process is binary. The models assume that potential adopters of an innovation either adopt or do not adopt the innovation.

(6) Sample is given only once to one customer.

The notations of the problem are shown below in the notations section.

2.2. Model Development. In Figure 1, the consumers are divided into two kinds: the individual adoptions and the multiple-unit adoptions; consumers can choose individual purchase or multiple-unit purchase when they just need the products; here $\omega$ represents the average volume of multipleunit adoptions according to statistical significance. The portion of the individual adoptions diffusion is based on the Bass diffusion model. Since the idea of owning multiple units is considered new by the consumers, the multiple acquisition can be viewed as an innovation; therefore, multipleunit adoptions can be viewed as a diffusion process that corresponds with the Bass diffusion model. 


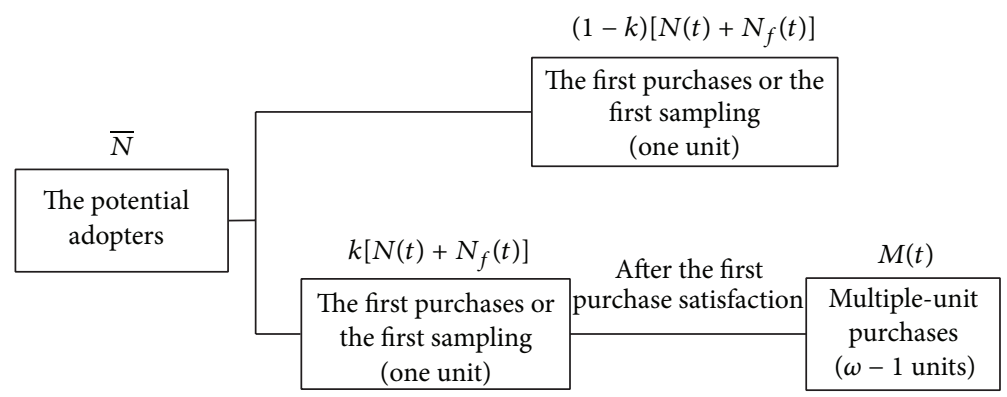

FIGURE 1: Diffusion pattern of new product incorporating individual and multiple-unit adoptions.

Accordingly, the potential multiple-unit adopters are $k N(t)$, which follows the idea of Steffens [6], and analogous to the Bass model, adoptions are driven by external influence and internal influence. So the fundamental diffusion model can be expressed as follows:

$$
\begin{gathered}
N(t+1)-N(t)=(a+b N(t))(\bar{N}-N(t)), \\
M(t+1)-M(t)=\left(a_{1}+b_{1} M(t)\right)(k N(t)-M(t)) .
\end{gathered}
$$

In (1), the initial values for $N(0)$ and $M(0)$, which respectively represents the volume of the new product adopter and the multiple-unit purchasers at time zero, have been assumed to be zero.

2.2.1. Impact of Sampling. Sampling is used to demonstrate a product's superiority and to get a potential customer to try the new product. Mahajan et al. [2] postulated that product sampling can be an effective way to create an initial pool of "adopters." The goal of product sampling for the firm is not only to initialize the diffusion process but also to improve brand loyalty. And during the diffusion process, the consumer can be divided into two groups: the group who gets the free samples and the group who purchases the product; that is,

$$
\begin{aligned}
N(t+1)-N(t)= & {\left[a+b\left(N(t)+N_{f}(t)\right)\right] } \\
& \times\left[\bar{N}-\left(N(t)+N_{f}(t)\right)\right], \\
M(t+1)-M(t)= & \left(a_{1}+b_{1} M(t)\right) \\
& \times\left[k\left(N(t)+N_{f}(t)\right)-M(t)\right],
\end{aligned}
$$

where $\gamma_{b} \gamma_{n}(t+1) n_{f}(t+1)=N_{f}(t+1)-N_{f}(t), \gamma_{n}(t+1)=$ $1-\left(N(t)+N_{f}(t)\right) / \bar{N}, N_{f}(0)=\gamma_{n}(0) n_{f}(0)$, and, especially, $\gamma_{n}(t)=1$ means all of the people getting the free samples at period $t$ have not adopted the product before.

Hence, the adopters include purchasers and the people who get the free sample. The sum of the adopters cannot exceed the upper limit of the potential adopters so that

$$
N(T)+N_{f}(T) \leq \bar{N}
$$

2.2.2. Sales Function. As discussed above, the adopters contain two parts: the consumers who insist on buying only one product and the consumers who insist on multiple adoption.
In the case of multiple-unit adoption, based on the research on multiple adoption by Steffens [6] and Tseng and $\mathrm{Hu}$ [14], $\omega$ means the average purchase amount of individual consumers in multiple adoption which includes the free one, so the adopters only pay for $\omega-1$ units products. Then the sales volume is

$$
\begin{aligned}
S(t+1)= & N(t+1)-N(t)+N_{f}(t+1)-N_{f}(t) \\
& +(\omega-1)(M(t+1)-M(t)) .
\end{aligned}
$$

2.2.3. Objective Function. One may also argue that product sampling is expensive for the firm, so it may not be economical to give free samples to every potential adopter. As a result, serious consideration must be given to the question of how many samples should be distributed. The firm needs to determine the "right" amount of sampling to avoid the unnecessary costs. Namely, one can get the objective function to maximize the net present value (NPV) of the firm given by

$$
\begin{gathered}
\pi=\sum_{t=1}^{T} \frac{1}{\left(1+i_{r}\right)^{t}}\left[(p(t)-c) S(t)-\left(h+c_{f}\right) n_{f}(t)\right] \\
-\left(h+c_{f}\right) n_{f}(0)+(p(0)-c) N_{f}(0),
\end{gathered}
$$

where the cost of handling the sample is $h$ per unit; it includes labor and material cost of wrapping, shipping, and handling the sample but does not include the cost of production $c . c_{f}(\leq$ $c)$ is the cost of experience sampling, and $p(t)$ is the price of the product at time $t$.

2.2.4. The Optimization Model. Taking multiunit ownership into consideration, a model given below can be established to provide an analytical framework for incorporating explicitly the effects of product sampling on the new product diffusion:

$$
\begin{aligned}
\max \pi & \\
\text { subject to } \quad N(t+1)-N(t) & =\left[a+b\left(N(t)+N_{f}(t)\right)\right] \\
& \times\left[\bar{N}-\left(N(t)+N_{f}(t)\right)\right], \\
M(t+1)-M(t) & =\left(a_{1}+b_{1} M(t)\right) \\
& \times\left[k\left(N(t)+N_{f}(t)\right)-M(t)\right], \\
N(T)+N_{f}(T) \leq & \bar{N}, \quad N(0)=0, M(0)=0 .
\end{aligned}
$$


In the model (6), the optimal levels of product sampling are the values of $F_{i}(t)\left(=n_{f_{i}}(t) \gamma_{b} / \bar{N}\right)(t=1,2, \ldots, T)$ at which the NPV achieves the maximum value. Although the model (6) specifies the optimization formulation to determine the optimal size of product sampling, and those variables cannot be integrated to generate an explicit closed form to determine $F_{i}(t)$. They, however, can be solved numerically to find the optimal values of $F_{i}(t)$ for given values of $a, a_{1}, b, b_{1}, \bar{N}$, $i_{r}, c$, and $h$. Assuming various values of these parameters, the model can be solved numerically later to obtain some flavor about the nature and role of product sampling on the diffusion process of multiple-unit ownership.

\section{Analysis of Product Sampling}

In the product diffusion process, the timing and the volume of sampling are very important decision variables for the firm. How to play the role of sampling policy and how the firm determines sampling rate to achieve the maximizing profits according to the actual situation would be discussed here. To facilitate comparative analysis, some parameters, referenced to the literature of Jain et al [8] and Mahajan et al. [2], are defined as follows.

(1) The coefficients of external influence: $a=0.02, a_{1}=$ 0.3 and the coefficients of internal influence: $b=0.35$, $b_{1}=0.35$.

(2) The potential number of ultimate adopters is $N=54$ million; the product cycle life: $T=40$.

(3) Discount rate $i_{r}=0.1$, and the price of the product under unchanged pricing strategy and its cost are: $p=\$ 10$ and $c=\$ 4$. When discussing the impact of sampling level, the cost of sampling $c_{f}=\$ 1$ and the handling cost $h=\$ 1$.

(4) The volume of multiple-unit purchase of the product $\omega=3$, and the ratio of multiple-unit adopters $k$ is 0.1 .

The potential market is assumed to be constant during the diffusion process and the firm's goal is to maximize the profit; hence the analysis of sampling's impact on the diffusion would be made. Corresponding to the different purchase behavior, individual adoption case $(k=0)$ and multiunits adoption case $(k \neq 0)$, the sampling level may be changed.

3.1. Case of $k=0$. When $k=0$, there is only individual adoption in the market, the objective of sampling is to initiate the diffusion process. In case of without sampling, the NPV is $\$ 142.89$ million, and the sales peak is gotten at period 10. The NPV is enhanced about $3.14 \%$, when there exists experience sampling, and the sales peak is brought forward by two periods (Figure 2). The optimal sampling time is at time zero and sampling level is 3.9\% (Table 1). All these manifest that sampling is an effective promotion tool to accelerate the diffusion process, and the comparative analysis of sampling shows that sampling at time zero can get higher NPV. Thus, a new product is being launched in the market; the firm needs to send few free samples to make the consumers realize the product, and the consumers would become the potential adopters.

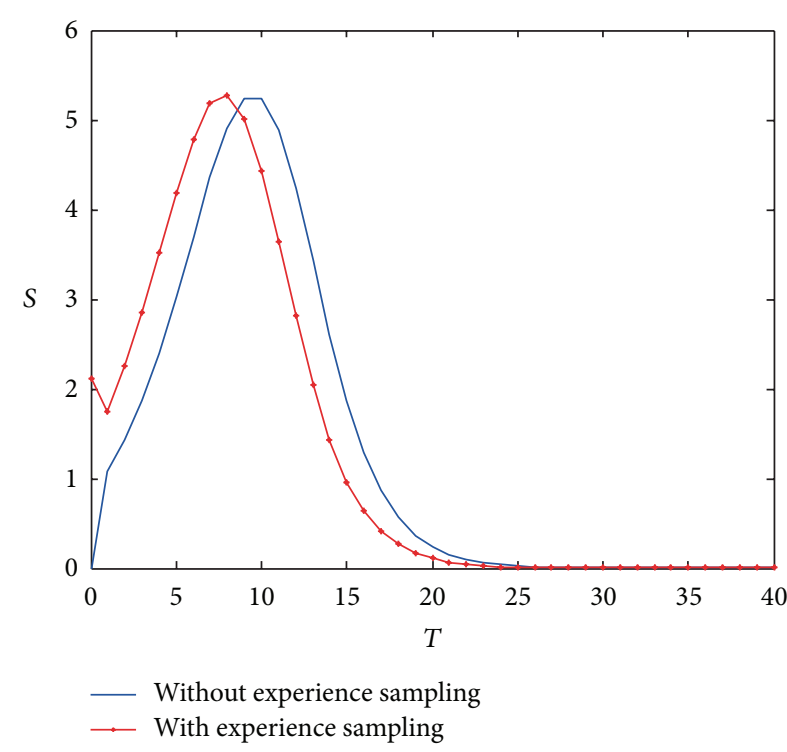

FIGURE 2: Impact of experience sampling on the sales curve.

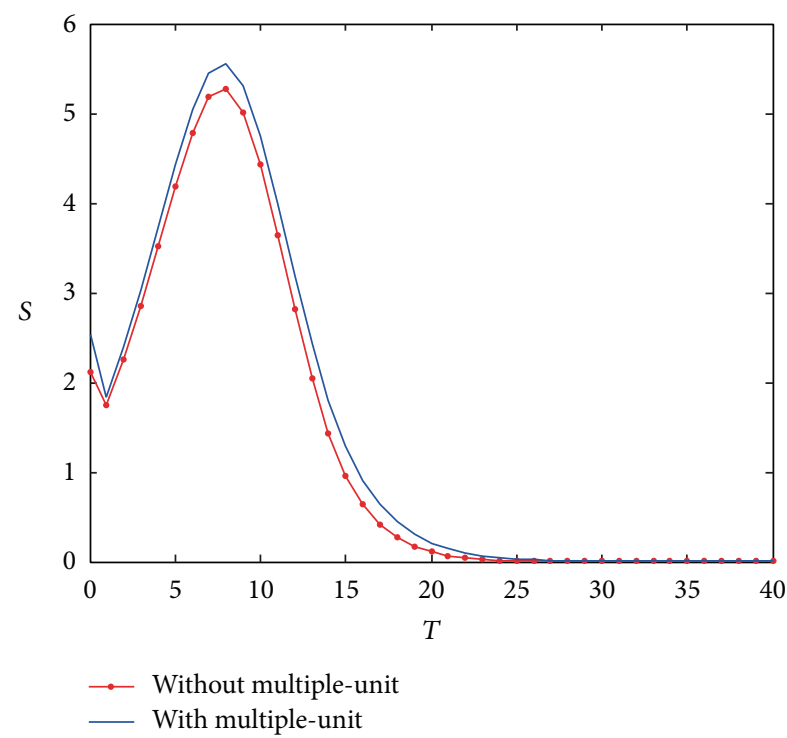

FIGURE 3: Impact of multiple-unit ownership on the sales.

3.2. Case of $k \neq 0$. The case of $k \neq 0$ means there is multipleunit adoptions in the whole diffusion process. In case of individual adoption, sampling promotes the diffusion and advances the sales volume peak period, thus increasing the firm's profit. Similarly, with multiunit purchase behavior, sampling speeds up the diffusion process, the optimal NPV is higher than the case of only individual adoption whether there is sampling or not (Table 1), and the extent of growth is between $5.7 \%$ and $8.2 \%$. Obviously, the optimal NPV is higher because of higher sales volume; the sales under multiple-unit purchase are $14 \%$ higher than sales under individual purchase (Figure 3). When the potential consumers are determined, customers with the ratio of $k$ choose to bulk-purchase, the multiunit purchase behavior can increase the impact of oral communication as well as single purchase, advance the sales 
TABLE 1: Comparison of NPV with or without experience sampling.

\begin{tabular}{lcccccc}
\hline \multirow{2}{*}{ Purchase behavior } & \multicolumn{2}{c}{ NPV } & \multicolumn{2}{c}{ The period of sales peak } & \multicolumn{2}{c}{ Sampling level } \\
& ID & MU & ID & MU & ID & - \\
\hline Without sampling & 142.89 & 164.53 & 10 & 10 & - \\
With sampling & 147.29 & 173.52 & 8 & 8 & $3.9 \%$ \\
\hline
\end{tabular}

ID: individual adoption; MU: multiunit adoption.

TABLe 2: Parameters and parameter level settings.

\begin{tabular}{lc}
\hline Parameters & Parameters level \\
\hline$a$ & $0.005,0.01,0.02,0.04,0.08$ \\
$a_{1}$ & $0.075,0.15,0.3,0.6,1.2$ \\
$b$ & $0.21,0.28,0.35,0.42,0.49$ \\
$b_{1}$ & $0.21,0.28,0.35,0.42,0.49$ \\
$k$ & $0,0.1,0.2,0.3$ \\
\hline
\end{tabular}

peak, and speed up the diffusion of the product and increase firms profits. At the final stage of the diffusion process, the adopters would be more with sampling, so sampling not only promotes the diffusion but also increases the sales volume.

\section{Sensitivity Analysis}

Model (6) is a nonlinear optimization problem; if the parameters are changed, the sampling level may be changed as well. Hyman [15] said that the acceptability of parameter estimation procedures is dependent upon the error sensitivity of these models. And Wang and Chang [16] conducted a research of efficient parameter estimation method for multiple generation product forecasting by using the hybrid genetic algorithm (HGA). For analyzing the stability of the optimal sampling level under different parameters, this section mainly analyzes the relationship between the parameters and the sampling level. The results would allow one to understand under which conditions of parameter values the NPV and sampling level could be the best.

4.1. Parameters Selection. The variables selected for the sensitivity analysis include continuous variables: the diffusion parameters (external coefficients $a, a_{1}$ and internal coefficients $b, b_{1}$ ) because the impact of different levels of corporate advertising, the innovation coefficients, and imitation coefficients will appear different. Considering the multiple-unit adoption diffusion, the ratio of multiple-unit adopters $(k)$ has a significant impact on the diffusion process. Sultan et al. [17] gave a meta-analysis of applications of diffusion models with 213 groups data in 15 papers shown below: for the analyses fitting on the equations above, the maximal coefficients of external influence is 0.23 and the minimal one is 0.00002 ; the maximal coefficients of external influence is 0.99 and the minimal one is 0.00003 . To have a comprehensive analysis of these parameters, the parameters and classification levels are shown in Table 2.

In total, there are $5^{4} * 4=2500$ treatments. For each experiment running, MATLAB would output the NPV and sampling level in time. To better know how much

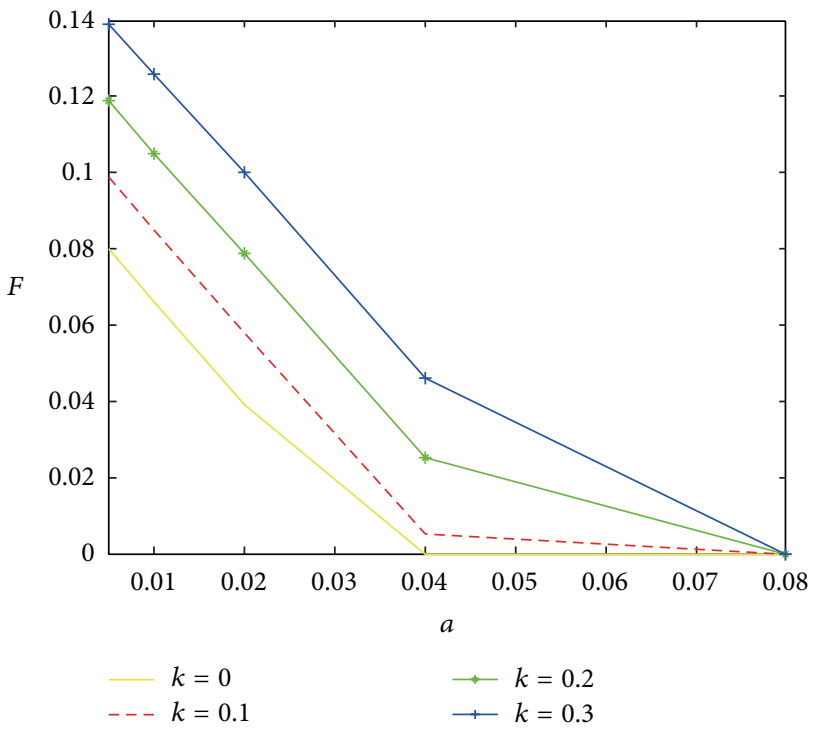

FIGURE 4: Impact of $a$ on sampling level.

the parameters affect the sampling level and to determine which parameters have greater influence on the diffusion, the following analysis would use statistical analysis to make better understanding in the relationship between the experimental parameters and the NPV and sampling level.

4.2. Local Sensitivity Analysis. With the increase of external coefficients, $a$, sampling level is declining and decreasing to $0 \%$ as the external influence is high enough (Figure 4). Given higher value for external coefficients, there would be enough innovators to adopt the product, and attempt to generate additional innovators through sampling may be a waste of resources. However, the sampling level increases along with the increase of the external coefficients of multiunit adoptions, $a_{1}$, and the rate of increase gradually decreases, when $a_{1}=1.2$, the sampling level is more than $6 \%$ (Figure 5). $a_{1}$ increase means that the idea of purchasing multiple-unit in the consumers is strong, since the consumer would choose whether to do a multiple-unit purchase after they try the product, so it deserved the firm to send more free samples to let the consumer try it. As shown already, four curves respectively express the sampling levels with different ratio of multiunit adopters. The more multiunit adopters, the more sampling worth sending; because of the higher sales volume when the consumers multiple-purchase, the marginal benefit of sampling is larger.

Figure 6 shows the influence of internal coefficients, $b$, on sampling level. If $k=0$ or $k=0.1$, with the increase 


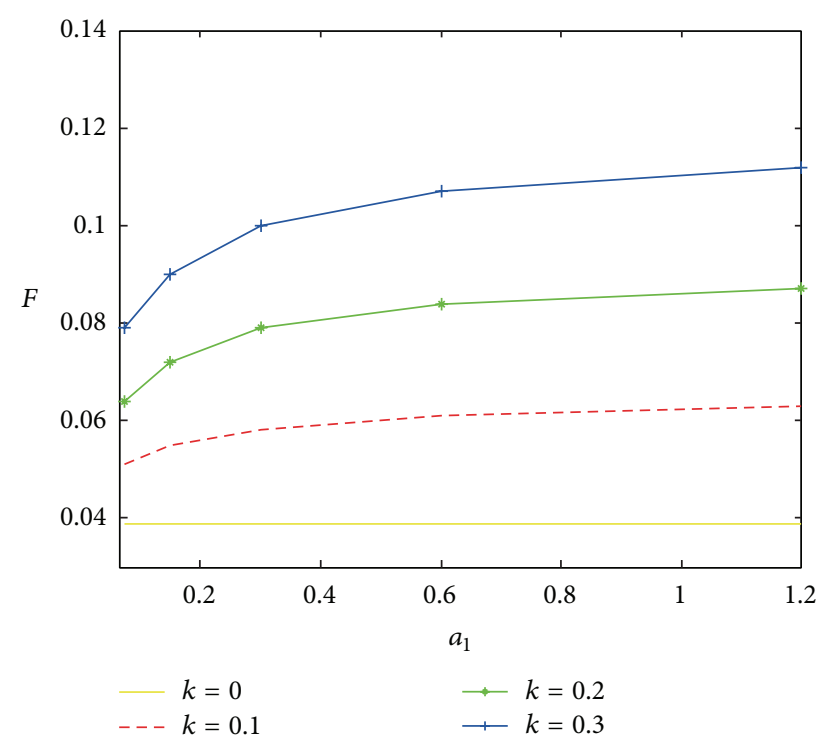

FigURE 5: Impact of $a_{1}$ on sampling level.

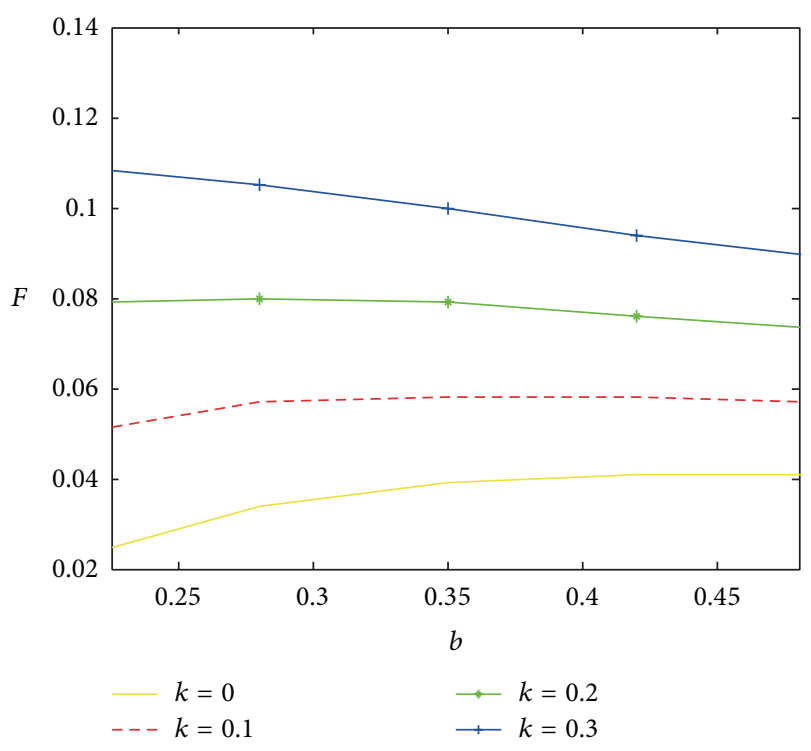

FIgURE 6: Impact of $b$ on sampling level.

of internal coefficients, sampling level is increasing at first, and when the internal influence increases to a certain value, the sampling level begins to drop. This situation means the oral communication effect increases as the internal influence increases; the firm needs more free samples to make consumers get a deeper realization of the product; and when the internal influence increases to a certain level, oral communication would promote the diffusion of product, and this time more sampling only increases the cost. However, when the multiunit adopters are more enough (like $k=0.2$ or $k=0.3$ ), the adopters could form a strong effect by oral communication and it would promote the product diffusion, so the sampling level is declining with the increase of internal coefficients. Similarly, $b_{1}$ represents the oral communication influence of multiunit adopters; it has little influence on the

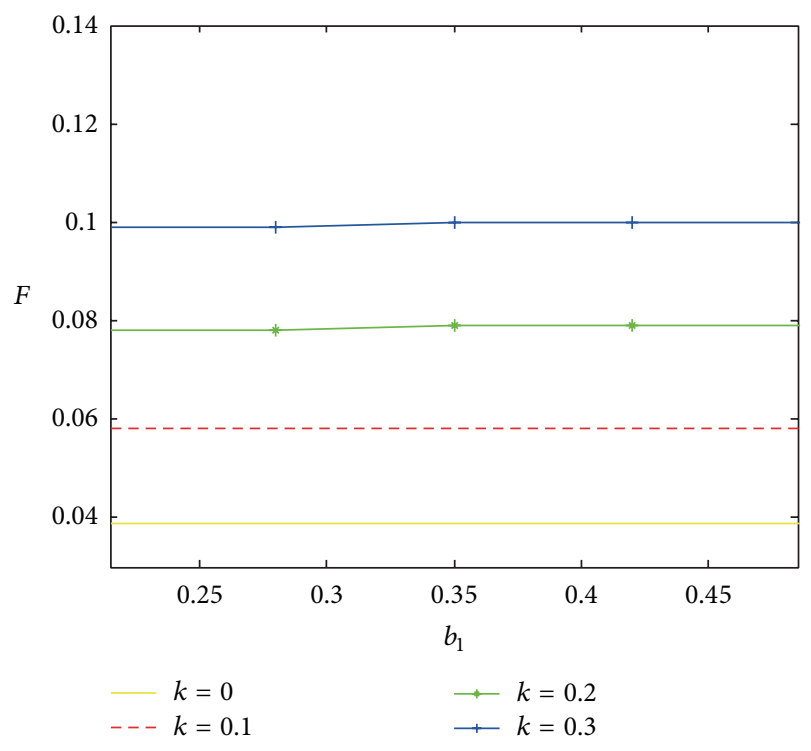

FIGURE 7: Impact of $b_{1}$ on sampling level.

sampling level (Figure 7). Because if the consumer choose to do a multiple-unit adoption, the communication among the multiunit adopters is so large that the free samples has little attractive.

4.3. Global Sensitivity Analysis. As opposed to local sensitivity analysis methods, Bolade and Pavel [18] said that global sensitivity analysis explores the full phase space of input parameters. To better develop further insight into the impact of every parameter on the whole model, this subsection turns to global sensitivity analysis and runs a full-factorial experimental design with five parameters at five levels each. Firstly, the regression analysis is shown with the experimental parameters as independent variables to analyze the impact of each parameter on NPV and sampling level through the $t$-statistic for its respective regression coefficients. Then removing the nonsampling sample cases, a linear regression analysis of the sampling level determination for samples cases is presented. The results show that the determination between the experimental parameters and dependent variables is greatly increased in this way.

4.3.1. Correlation Analysis. Pearson correlation coefficients test for testing the correlation between the parameters and NPV and sampling level is shown in Table 3. (1) Product external coefficients $a$ and internal coefficients $b$ have a positive influence on the NPV; however, both of them have a negative influence on sampling level. (2) For coefficients $b_{1}$, it is not significant with NPV and sampling level because the $P$ value equals 0.7069 and 0.8493 , which exactly corresponds to Figure 7. (3) Variables of $a, a_{1}$, and $b$ are significant at $P<0.05$ which shows the strong correlation between the parameters $a$, $a_{1}$, and $b$ and the product sampling level.

Thus, using all parameters as independent variables, two linear regression models are fitted when considering the sampling level and NPV as dependent variables, respectively, 
TABLE 3: The correlation test with test parameters and decision variables.

\begin{tabular}{lcccccrr}
\hline & \multicolumn{7}{c}{ Pearson correlation coefficients, $N=2500$ Prob. $>|r|$ under $H_{0}: \rho=0$} \\
& $a$ & $a_{1}$ & $b$ & $b_{1}$ & $k$ & NPV \\
\hline NPV & 0.72321 & 0.12682 & 0.59239 & 0.00752 & 0.06249 & 1 & -0.71171 \\
$P$ value & $<0.0001$ & $<0.0001$ & $<0.0001$ & 0.7069 & 0.0018 & $<0.0001$ \\
\hline sl & -0.75028 & -0.12462 & -0.05276 & 0.0038 & 0.02669 & -0.71171 \\
$P$ value & $<0.0001$ & $<0.0001$ & 0.0083 & 0.8493 & 0.1822 & $<0.0001$ \\
\hline
\end{tabular}

based on the 2500 experiment data sets. Tables 4 and 5 present the $t$-value and the significance levels to summarize the level and direction of the particular variable's influence. The significant levels $(P<0.0001)$ and the adjusted $R$-squares (0.5811 and 0.9305) indicated that the effect of interaction between them is relative strong.

Given by their $t$-values, parameters are ordered as $a>$ $a_{1}>b>k$ with respect to their influences on the sampling level differences. With respect to NPV level, the ordering is $b>a>\mathrm{sl}>a_{1}>k$. This ordering indicates that in the diffusion process, the external influence of the product and the ratio of multiple-unit adopters are the two most influential parameters to influence the NPV and sampling level. For $k=0$, the sampling level and NPV equal 3.9\% and \$147.29 million, respectively (shown in Table 1). When considering the multiunit purchase $(k=0.1)$, the sampling level and NPV are 5.8\% and $\$ 173.52$ million, which confirms that the ratio of multiunit adopters has an obvious influence on both sampling level and NPV just as the shown orderings.

4.3.2. Logistic Regression. Actually, among 2500 sets of experimental data, 1410 sets of them need sampling, and others do not need it, so the above regression models cannot be sufficient to fully explain the sampling level differences. To make more accurate analysis, logistic regression analysis is first used to distinguish whether sampling is needed, then linear regression is discussed for the needed sampling cases.

Here sampling or not could be a discrete variable as dependent variable of logistic regression model, defining a dependent variable, sampling level (sl), which means sampling level is to be 1 if sampling is needed and to be 0 otherwise. To use the logistic regression model for discrimination analysis, compute an estimate of the probability that sl is equal to 1 given the values of the predictor variables to decide whether sampling is needed. If the estimated probability is less than 0.5 , the sampling is needed.

The chi-square test in Table 6 indicates that the predictor variables being used are statistically significant predictors. The test considers all predictors variables simultaneously.

Logistic regression analysis gives a probability to decide whether sampling or not; Table 7 contains the classification summary matrix for 2500 sets of data; the column of sl dummy and the labels on the top show the groups whether sampling or not. It can be seen that $97.71 \%$ of the data without sampling would be classified correctly by the logistic discriminant rule and that 1400 of the $99.29 \%$ sets of data with sampling would be classified correctly.

According to Jassim [19], logistic regression has less stringent requirements than linear regression models in
TABLE 4: Statistics of multiple linear regression for sampling level.

\begin{tabular}{lccc}
\hline Variable & Parameter estimate & $t$-value & $\{$ Pr. $>|t|\}$ \\
\hline Intercept & 0.04713 & 28.55 & $<0.0001$ \\
$a$ & -0.63628 & -57.95 & $<0.0001$ \\
$a_{1}$ & -0.00705 & -9.63 & $<0.0001$ \\
$b$ & -0.01233 & -4.08 & $<0.0001$ \\
$b_{1}$ & 0.00088857 & 0.29 & 0.769 \\
$k$ & 0.00552 & 2.06 & 0.0394 \\
\hline \multicolumn{4}{c}{}
\end{tabular}

TABLE 5: Statistics of multiple linear regression for NPV.

\begin{tabular}{lccc}
\hline Variable & Parameter estimate & $t$-value & $\{$ Pr. $>|t|\}$ \\
\hline Intercept & 69.12142 & 78.04 & $<0.0001$ \\
sl & -338.33958 & -36.28 & $<0.0001$ \\
$a$ & 485.81727 & 62.01 & $<0.0001$ \\
$a_{1}$ & 5.81241 & 16.74 & $<0.0001$ \\
$b$ & 154.06014 & 108.97 & $<0.0001$ \\
$b_{1}$ & 2.31058 & 1.64 & 0.1012 \\
$k$ & 16.64732 & 13.33 & $<0.0001$ \\
\hline \multicolumn{4}{c}{}
\end{tabular}

TABLE 6: Testing global null hypothesis.

\begin{tabular}{lccc}
\hline Test & Chi-square & DF & Pr. $>$ Chi-sq. \\
\hline Likelihood ratio & 3264.5956 & 5 & $<0.0001$ \\
Score & 1740.5363 & 5 & $<0.0001$ \\
Wald & 58.8774 & 5 & $<0.0001$ \\
\hline
\end{tabular}

TABLE 7: Sampling level dummy by estimation.

\begin{tabular}{|c|c|c|c|}
\hline \multirow{2}{*}{ sl dummy } & \multicolumn{3}{|c|}{ Estimation } \\
\hline & No & Yes & Total \\
\hline \multirow[t]{4}{*}{0} & 1065 & 25 & 1090 \\
\hline & 42.6 & 1 & 43.6 \\
\hline & 97.71 & 2.29 & \\
\hline & 99.07 & 1.75 & \\
\hline \multirow[t]{4}{*}{1} & 10 & 1400 & 1410 \\
\hline & 0.4 & 56 & 56.4 \\
\hline & 0.71 & 99.29 & \\
\hline & 0.93 & 98.25 & \\
\hline \multirow[t]{2}{*}{ Total } & 1075 & 1425 & 2500 \\
\hline & 43 & 57 & 100 \\
\hline
\end{tabular}


TABLE 8: Analysis of maximum likelihood estimates.

\begin{tabular}{lccccc}
\hline Parameter & DF & Estimate & Standard error & WaldChi-square & Pr. $>$ Chi-Sq. \\
\hline Intercept & 1 & -23.3684 & 79.4137 & 0.0866 & 0.7686 \\
$a$ & 1 & 1634.3 & 3971.2 & 0.1694 & 0.6807 \\
$a_{1}$ & 1 & 6.2189 & 0.8759 & 50.4123 & $<0.0001$ \\
$b$ & 1 & -49.752 & 6.6349 & 56.228 & $<$ \\
$b_{1}$ & 1 & $7.19 E-13$ & 2.0161 & 0 & 0.0001 \\
$k$ & 1 & -3.1812 & 1.8172 & 3.0646 & 0.08 \\
\hline
\end{tabular}

TABLE 9: Statistics of multiple linear regression for sampling level for selected sampling experiments.

\begin{tabular}{lccc}
\hline Variable & Parameter estimate & $t$-value & Pr. $>|t|$ \\
\hline Intercept & 0.08206 & 89.03 & $<0.0001$ \\
$a$ & -2.76414 & -102.39 & $<0.0001$ \\
$a_{1}$ & -0.01118 & -27.35 & $<0.0001$ \\
$b$ & -0.0302 & -17.96 & $<0.0001$ \\
$b_{1}$ & 0.00158 & 0.96 & 0.3357 \\
$k$ & 0.00719 & 4.96 & $<0.0001$ \\
\hline \multicolumn{4}{c}{} \\
\hline
\end{tabular}

TABLE 10: Statistics of multiple linear regression for NPV for selected sampling experiments.

\begin{tabular}{lccc}
\hline Variable & Parameter estimate & $t$-value & Pr. $>|t|$ \\
\hline Intercept & 48.16522 & 26.67 & $<0.0001$ \\
sl & -143.90929 & -7.1 & $<0.0001$ \\
$a$ & 831.21517 & 13.92 & $<0.0001$ \\
$a_{1}$ & 3.11781 & 8.11 & $<0.0001$ \\
$b$ & 188.27021 & 132.86 & $<0.0001$ \\
$b_{1}$ & 2.16618 & 1.74 & 0.0818 \\
$k$ & 6.12911 & 5.52 & $<0.0001$ \\
\hline \multicolumn{4}{c}{}
\end{tabular}

general, but it requires the observations to be independent and the independent factors are linearly related to the logit of the response variable. To show how the variables affect the probability, Table 8 lists the parameter estimates of the parameters in the logit function:

$$
\begin{aligned}
\mathrm{SL}= & -23.3684+1634.3 a-6.2189 a_{1}-49.752 b \\
& +(7.19 E-13) b_{1}-3.1812 k .
\end{aligned}
$$

Putting the original parameters $\left(a=0.02, a_{1}=0.3, b=\right.$ $b_{1}=0.35, k=0$, or $k=0.1$ ) into the logit function, the estimated probability of individual adoption and multiunit adoption is 0.00197 and 0.00143 , respectively, which is far less than 0.5 , indicating that whether with or without multiunit adopters, sampling is needed.

4.3.3. Linear Regression for Sampling. Based on the discussion above, the 1410 sampling experiments are taken out from the total 2500 experiments to do a multiple linear regression, considering the sampling level and NPV as dependent variables, respectively. Tables 9 and 10 are the statistics of multiple linear regression for selected sampling experiments and NPV, showing that $a, a_{1}, b$, and $k$ variables in the regression model are significant at $P<0.0001$, which confirms the above results.

(1) The adjusted $R$-square increases significantly from 0.5811 to 0.8934 , which illustrates that the relevance of the parameters and the sampling level has improved a lot and the adjusted model fits the sampling level very well. Meanwhile, through the statistics analysis of NPV with the 1815 selected experiments, the adjusted $R$-square also increases from 0.9305 to 0.9538 , which suggest that the selected experiments have little influence on the NPV.

(2) The values of $a$ and $b$ are negative and $a_{1}, k$ are positive, so with the value of $a$ and $b$ increasing, sampling level will decrease, and increasing $a_{1}, k$, sampling level will increase, which is the same as local sensitivity analysis. Due to the $P$ value of $b_{1}$ being 0.3357 , the value of $b$ is not significant with NPV and sampling; this data are more convincing to show that this model fits the sampling data very well.

(3) The ratio of predicted residual SS (0.05244) and sum of squared residuals (0.05196) is 1.0092 , approaching 1 , which also indicates that the adjusted model is more accurate than the earlier one.

(4) The regression model for sampling level being as dependent variable is as follows:

$$
\begin{aligned}
\mathrm{SL}= & 0.08206-2.76414 a+0.01118 a_{1} \\
& -0.0302 b+0.00719 k .
\end{aligned}
$$

After substituting the parameters $\left(a=0.02, a_{1}=0.3, b=\right.$ $b_{1}=0.35, k=0$, or $k=0.1$ ) into the adjusted sampling level regression model, the adjusted model fits the sampling level much better. Through GLM, it also can predict the sampling level with 95\% confidence interval (Table 11).

All the conclusions illustrate that it is necessary for a firm to conduct logistic analysis to decide whether sampling or not; if sampling is needed, then use the adjusted regression models to get the sampling levels. The discussion above presents a two-stage method to improve the accuracy of making sample level, including several operational steps shown as follows.

Step 1. Find a similar model based on product-related parameters and then fit the diffusion equations.

Step 2. Get the parameters according to the parameter ranges and compute the simulated data under different situations.

Step 3. Make statistical analysis for all obtained simulated data and then take the relevant parameters into logistic 
TABLE 11: The GLM procedure results for sampling level.

\begin{tabular}{lcccccccccc}
\hline$a$ & $a_{1}$ & $b$ & $b_{1}$ & $k$ & Predicted & Std. Err. & LCL & UCL & LCLM & UCLM \\
\hline 0.02 & 0.3 & 0.35 & 0.35 & 0.1 & 0.014123 & 0.000302501 & 0.002179189 & 0.026067 & 0.013530 & 0.014717 \\
\hline
\end{tabular}

regression to see whether sampling or not, which is called stage one.

Step 4. If it is worth sampling, then take these parameters into adjusted sampling level model to determine the accurate sampling level, which is called stage two.

\section{Conclusion}

Multiple-unit ownership of nondurable products is an important component of sales in many product categories. However, little attention has been paid to models for multiple-unit ownership in the diffusion literature, specially, the studies of promotion strategies in the model have gotten little attention. This paper, based on the idea of Steffens [6], established an optimization model for multiple-unit ownership, which characterizes the multiple-unit adoptions as a diffusion process, and has done a sensitive analysis and got some key findings. (1) Comparing the sampling and nonsampling tactics, the appropriate experience sampling could accelerate the diffusion process and advance the sales peak period, resulting in the profit increase. The best time to carry out the strategy is as soon as possible. (2) Compared to the diffusion process under individual purchasing behavior, multiple-unit purchasing behavior can increase sales to create more profit, and it deserves sending more samples. (3) The external influence and multiunit rate are the two most important parameters to influence the sampling level and NPV of the new products in the diffusion process. (4) Through the global sensitivity analysis, a two-stage method is more widely fitted to estimate the relevant parameters in the case of parameters lack of accuracy and to be able to construct a 95\% confidence interval for the predicted sampling level.

Above all, the paper first solved the optimum sampling level and then used the statistical methods to analyze the sampling trend and verify the optimum sampling trend. The main contribution of this study lies in presenting a two-stage method to analyze whether sampling or not, if sampling, how much should be sent out. So some important managerial benefits of the model and the results are implied. (1) When the firm launches a new product, product sampling is an effective way to promote the product, especially the low-value consumable products that can be multiple-purchased. (2) It's better for the firm to use free samples before the products being launched in the market. (3) Before making a decision, the firm needs to do a statistical analysis of the parameters of the products, since parameter combination has an impact on the optimal sampling level.

For above results, these findings can be easily applied into the fast moving consumer goods, but how to use these in reality is a tough issue; the implementation of the model involves following steps: (1) identification of the parameters in the model, such as coefficients of external influence, coefficients of internal influence, and market potential: this can be obtained by getting the historical data and statistical analysis from some consulting companies and market research companies, (2) specification of the discount rate: although the commonly used yearly discount rate is $10 \%$, it depends on the economic condition in reality. Before launching the new product, the sales manager should determine a feasible discount rate, (3) determination of the price of the new product and estimation of the product's unit cost and handling cost: it is a part of the firm's marketing plan, as price is one of the key marketing-mix variables, which can be referred to the prices of similar products or related product prices, and (4) simulation of the parameters and data in the model to obtain the optimal sampling level and give the management suggestions.

A main limitation of above discussion is that the consumers purchasing behavior does not change over the whole diffusion process and the definition of new nondurable product is too ideal to limit the discussion and application. Also, the paper only considered the free samples having positive effects, so an interesting direction of research would be to expand the model by reflecting the negative effects or mixed ones.

\section{Notation}

$t$ : $\quad$ Time (from 1 to $T$ )

$\bar{N}$ : The number of potential adopters

$a$ : Coefficients of external influence of the product

$b$ : Coefficients of internal influence of the product

$a_{1} / b_{1}$ : Coefficients of external influence/internal influence on the subsequent multiple-unit adoptions

$N(t)$ : The cumulative number of adopters who purchase the product by time $t$

$M(t)$ : The cumulative number of multiple-unit adoptions by time $t$

$N_{f}(t)$ : The cumulative number of adopters by time $t$ who get the free sample of the product but never adopted the product before

$n_{f}(t)$ : The noncumulative number of the people who get the free sample of the product in period $t$

$S_{i}(t)$ : Sales volume of individual purchase of the product at time $t$

$S_{m}(t)$ : Sales volume of multiple-unit purchase of the product at time $t$

$F(t)$ : Noncumulative sampling level of the product at time $t$ 

$\gamma_{n}(t)$ : The nonadopter ratio of the people who get the free sample at time $t$
$\gamma_{b}$ : The ratio of potential adopters of the product in whole market
$\omega$ : Number of multiple-unit purchase of the product
$k$ : The ratio of multiple-unit adopters.

\section{Conflict of Interests}

The authors declare that there is no conflict of interests regarding the publication of this paper.

\section{Acknowledgment}

This research is supported by National Natural Science Foundation of China (Grant no. 70971091).

\section{References}

[1] F. M. Bass, "A new product growth for model consumer durables," Management Science, vol. 50, no. 12, pp. 1825-1832, 2004.

[2] V. Mahajan, E. Muller, and F. M. Bass, "New-product diffusion models," Handbooks in Operations Research and Management Science, vol. 5, pp. 349-408, 1993.

[3] R. Peres, E. Muller, and V. Mahajan, "Innovation diffusion and new product growth models: a critical review and research directions," International Journal of Research in Marketing, vol. 27, no. 2, pp. 91-106, 2010.

[4] B. T. Ratchford, S. K. Balasubramanian, and W. A. Kamakura, "Diffusion models with replacement and multiple purchases," in New-Product Diffusion Models, V. Mahajan, E. Muller, and Y. Wind, Eds., pp. 123-143, Kluwer Academic Publishing, Boston, Mass, USA, 2000.

[5] B. L. Bayus, S. Hong, and R. P. Labe Jr., "Developing and using forecasting models of consumer durables. The case of color television," The Journal of Product Innovation Management, vol. 6, no. 1, pp. 5-19, 1989.

[6] P. R. Steffens, "A model of multiple-unit ownership as a diffusion process," Technological Forecasting and Social Change, vol. 70, no. 9, pp. 901-917, 2003.

[7] Y. H. Wang, S. L. Yang, W. Y. Qian, and X. Z. Li, "Forecasting new product diffusion using grey time-delayed verhulst model," Journal of Applied Mathematics, vol. 2013, Article ID 109614, 6 pages, 2013.

[8] D. Jain, V. Mahajan, and E. Muller, "An approach for determining optimal product sampling for the diffusion of a new product," The Journal of Product Innovation Management, vol. 12, no. 2, pp. 124-135, 1995.

[9] B. Lammers, "The effect of free samples on the immediate consumer purchase," Consumer Marketing, vol. 8, no. 2, pp. 31-37, 1991.

[10] Z. N. Hu, B. Li, and J. P. Xu, "Impact of product sampling for diffusion," Systems Engineering Theory and Practice, vol. 33, no. 5, pp. 1192-1199, 2013.

[11] Z. N. Hu, "Incorporating price in optimal product sampling for diffusion," International Journal of Management Science and Engineering Management, vol. 1, no. 2, pp. 119-136, 2006.

[12] A. Heiman, B. McWilliams, Z. Shen, and D. Zilberman, "Learning and forgetting: modeling optimal product sampling over time," Management Science, vol. 47, no. 4, pp. 532-546, 2001.
[13] S. P. Li and Z. Jin, "Global dynamics analysis of homogeneous new products diffusion model," Discrete Dynamics in Nature and Society, vol. 2013, Article ID 158901, 6 pages, 2013.

[14] F.-M. Tseng and Y.-C. Hu, "Quadratic-interval Bass model for new product sales diffusion," Expert Systems with Applications, vol. 36, no. 4, pp. 8496-8502, 2009.

[15] M. R. Hyman, "The timeliness problem in the application of bass-type new product-growth models to durable sales forecasting," Journal of Business Research, vol. 16, no. 1, pp. 31-47, 1988.

[16] F.-K. Wang and K.-K. Chang, "Modified diffusion model with multiple products using a hybrid GA approach," Expert Systems with Applications, vol. 36, no. 10, pp. 12613-12620, 2009.

[17] F. Sultan, J. U. Farley, and D. R. Lehmann, "A meta-analysis of applications of diffusion models," Journal of Marketing Research, vol. 27, pp. 70-77, 1990.

[18] A. A. Bolade and M. B. Pavel, "Computational method for global sensitivity analysis of reactor neutronic parameters," Science and Technology of Nuclear Installations, vol. 2012, Article ID 109614, 11 pages, 2012.

[19] N. H. Jassim, "Sensitivity analysis to select the most influential risk factors in a logistic regression model," International Journal of Quality, Statistics, and Reliability, vol. 2008, Article ID 471607, 10 pages, 2008. 


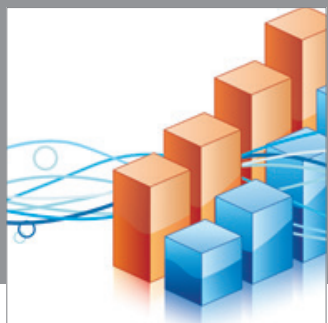

Advances in

Operations Research

mansans

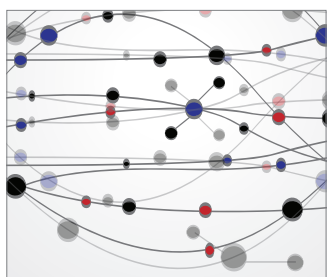

The Scientific World Journal
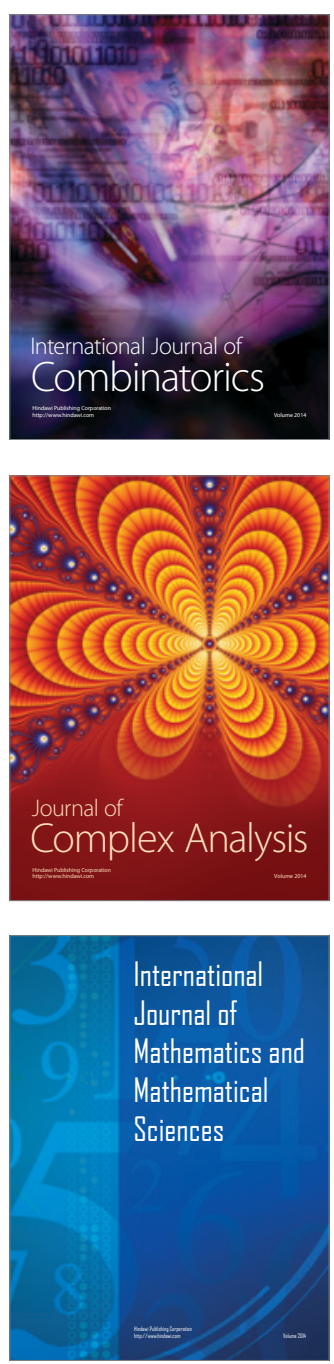
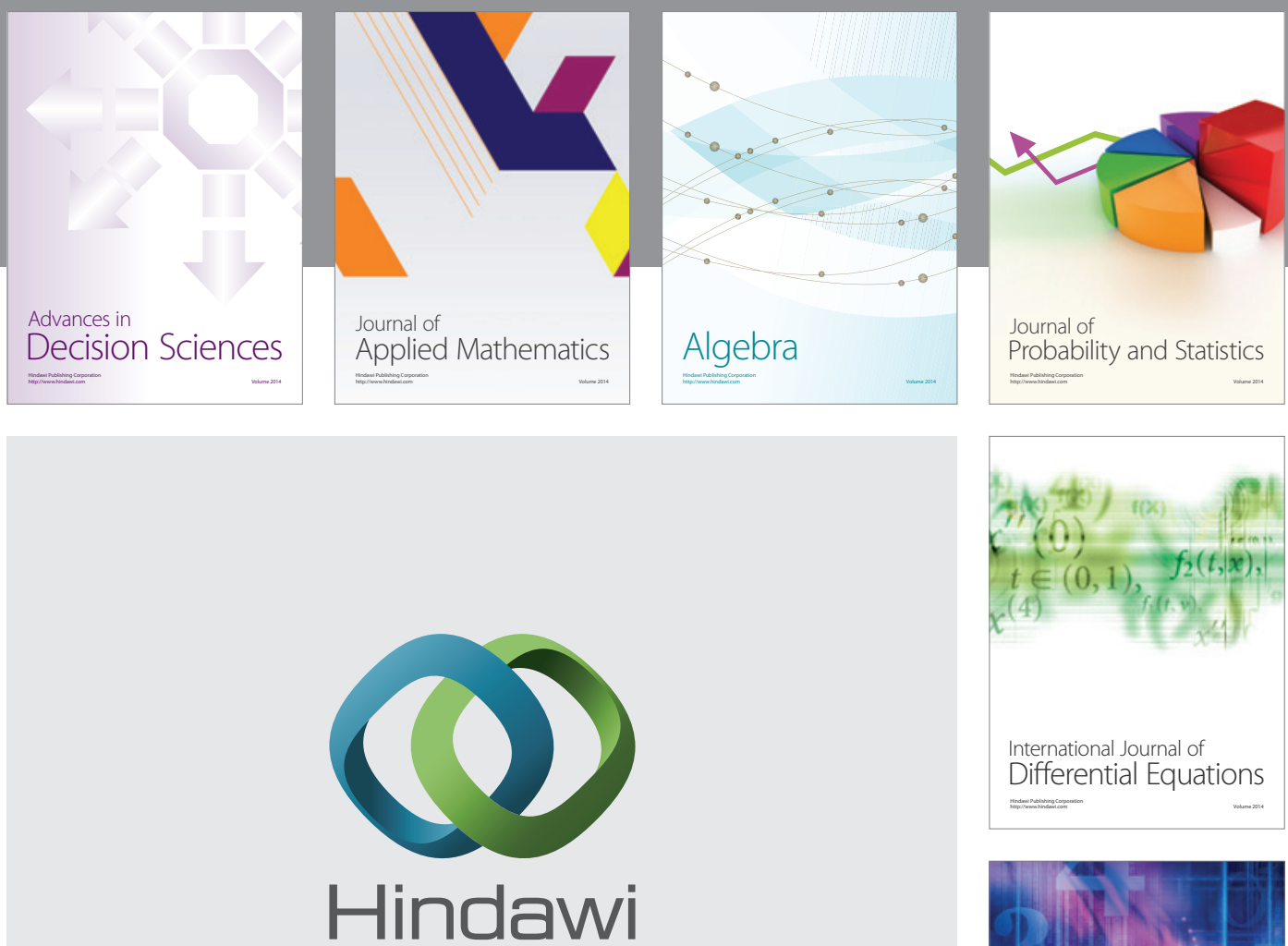

Submit your manuscripts at http://www.hindawi.com
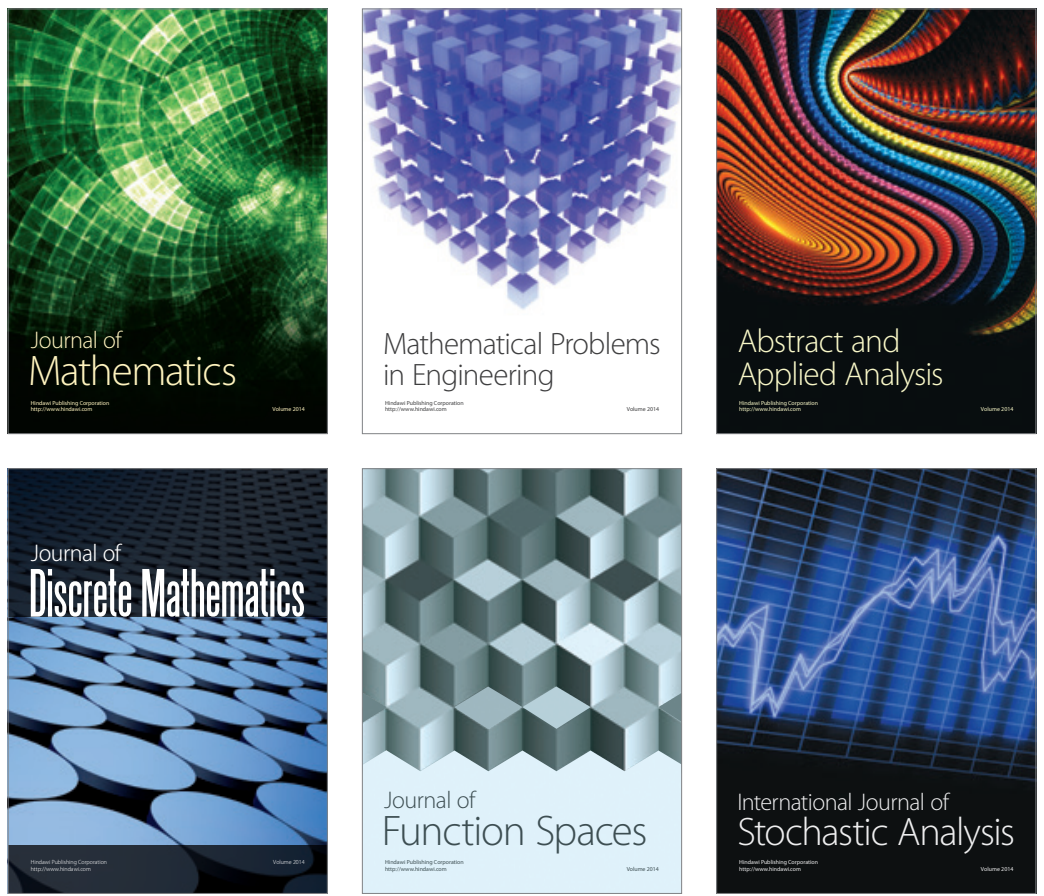

Journal of

Function Spaces

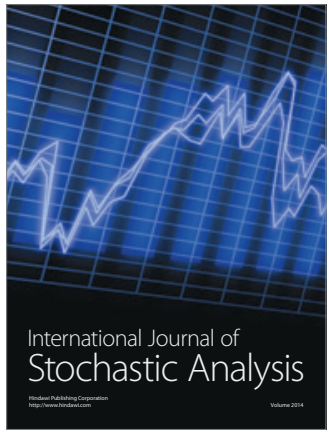

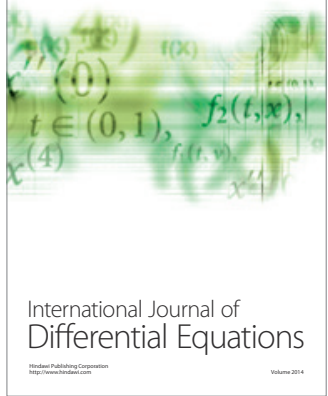
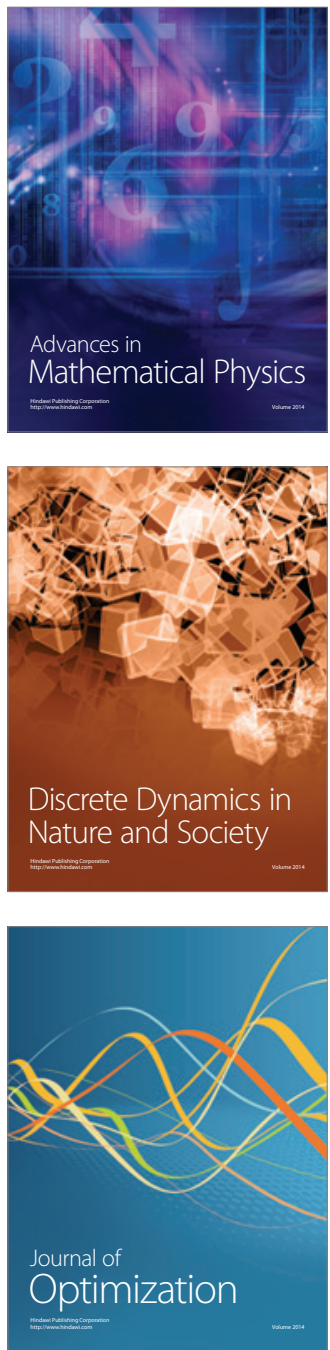